\title{
Experiencia de drenaje biliar percutáneo en pacientes del Hospital Ángeles Mocel
}

\author{
Experience of percutaneous biliary drainage in \\ patients at the Hospital Ángeles Mocel
}

\author{
Ricardo Santillán Morales, * José Luis Ríos Reina ${ }^{\ddagger}$ \\ Citar como: Santillán MR, Ríos RJL. Experiencia de drenaje biliar percutáneo en pacientes del Hospital \\ Ángeles Mocel. Acta Med. 2021; 19 (1): 15-20. https://dx.doi.org/10.35366/98564
}

\section{Resumen}

Introducción: El drenaje biliar percutáneo es una opción para tratamiento de ictericia obstructiva en quienes no es exitosa la colangiopancreatografía retrógrada endoscópica (CPRE). Objetivo: Presentar la experiencia del drenaje biliar percutáneo en pacientes con obstrucción de vía biliar del HAMo. Material y métodos: Se revisaron los expedientes de todos los pacientes que durante siete años fueron sometidos a drenaje biliar percutáneo por obstrucción benigna o maligna de vía biliar, obteniéndose información demográfica, presentación clínica, método y paraclínicos diagnósticos, causa, tasa de dilatación exitosa y de complicaciones asociadas. Resultados: Se identificaron 67 casos de pacientes sometidos a drenaje biliar percutáneo, la mayoría $(n=45 ; 65.7 \%)$ se presentaron entre los 40 y 60 años de edad. Tuvieron ictericia, pérdida de peso o fiebre el $85.7,77.6$ y $34.3 \%$, respectivamente. El $86.3 \%$ de los pacientes tuvieron lesiones malignas y el $13.4 \%$ benignas. La tasa de éxito de dilatación fue de $96.5 \%$; los no exitosos se debieron a múltiples adherencias o lesiones pancreáticas (3.5\%). Las complicaciones asociadas al procedimiento fueron: sepsis (3.6\%) y hemorragia (1.8\%). Conclusiones: El drenaje biliar percutáneo presenta una alta tasa de éxito en el HAMo y se asocia con una baja frecuencia de complicaciones.

Palabras clave: Drenaje biliar percutáneo, ictericia, obstrucción biliar, tasa de éxito.

\section{Abstract}

Introduction: Percutaneous biliary drainage is an option for treatment of obstructive jaundice in patients where endoscopic retrograde cholangiopancreatography is not successful. Objective: To present the experience of percutaneous biliary drainage in patients with bile duct obstruction. Material and methods: The records of all patients who underwent percutaneous biliary drainage due to benign or malignant bile duct obstruction were reviewed, obtaining demographic information, clinical presentation, diagnostic method and paraclinical tests, cause, rate of successful dilation and associated complications. Results: 67 cases of patients undergoing percutaneous biliary drainage were identified, the majority $(n=45 ; 65.7 \%)$ presented between 40 and 60 years of age. They had jaundice, weight loss or fever $85.7 \%, 77.6 \%$ and $34.3 \%$, respectively. Of these patients, $86.3 \%$ had malignant lesions and $13.4 \%$ benign. The dilation success rate was $96.5 \%$; the unsuccessful ones were due to multiple adhesions or pancreatic lesions (3.5\%). Complications associated with the procedure were: sepsis $(3.6 \%)$ and hemorrhage $(1.8 \%)$. Conclusions: Percutaneous biliary drainage has a high success rate in HAMo and is associated with a low frequency of complications.

Keywords: Percutaneous biliary drainage, jaundice, biliary obstruction, success rate.
* Médico Residente de Radiología e Imagen. Facultad Mexicana de Medicina de la Universidad La Salle. CDMX, México.

‡ Jefe de Imagenología, Hospital Ángeles Mocel. CDMX, México.

Correspondencia:

Ricardo Santillán Morales

Correo electrónico: dr_santillanricardo@outlook.com

\section{Abreviaturas:}

CPRE $=$ Colangiopancreatografía retrógrada endoscópica.

$\mathrm{DBP}=$ Drenaje biliar percutáneo.

$\mathrm{Fr}=$ French.

$\mathrm{HAMo}=$ Hospital Ángeles Mocel.

Aceptado: 29-01-2020.

www.medigraphic.com/actamedica 


\section{INTRODUCCIÓN}

La ictericia obstructiva es la consecuencia de la estasis biliar causada por la obstrucción del flujo biliar por cálculos, tumores o inflamación y es uno de los padecimientos que con alta frecuencia afecta las vías biliares. ${ }^{1}$

El manejo de elección del tratamiento de la ictericia obstructiva es la colangiopancreatografía retrógrada endoscópica (CPRE), tanto en pacientes con tumores malignos no resecables, como en pacientes con patologías benignas como estenosis postoperatorias, colangitis esclerosante primaria y postrasplantados de hígado. ${ }^{2,3}$

Sin embargo, la tasa de falla de la CPRE es de $10-30 \%$ con importantes variaciones entre series; otros reportan una tasa de falla de la CPRE de $3-10 \%{ }^{4}$

En pacientes en quienes la CPRE falla, el drenaje biliar percutáneo (DBP) es una alternativa altamente efectiva, que logra un éxito mayor al 90\% cuando los conductos biliares están dilatados, y del $70 \%$ con conductos de diámetro normal. ${ }^{5-7}$

Otras indicaciones del DBP incluyen descompresión de una obstrucción baja no resecable, descompresión previa a la colocación de una prótesis percutánea, tratamiento combinado percutáneo-CPRE, y tratamiento transyeyunal de estenosis benignas o malignas, entre otros. ${ }^{8}$

No obstante, a pesar de la utilidad del DBP, también se presentan complicaciones en una proporción diversa de pacientes; varía la tasa de complicaciones y se han reportado en 4.6-25\% de los pacientes sometidos al procedimiento; incluso algunos estudios han reportado mayor frecuencia. ${ }^{9,10}$

En el presente trabajo se presenta la experiencia de realización de drenaje biliar percutáneo en pacientes con obstrucción de vía biliar del Hospital Ángeles Mocel.

\section{MATERIAL Y MÉTODOS}

Para los procedimientos se empleó la sala de hemodinamia con equipo de la marca Siemens Megalix Cat Plus serie 50075.

Previa valoración por el Servicio de Anestesiología, en todos los casos los pacientes se encontraban en rangos de la normalidad en titulación de tiempos de coagulación e índice internacional normalizado (INR), utilizando antibiótico endovenoso, previo a la punción, siendo 1 g IV dosis única de ceftriaxona, como profilaxis.
Figura 1:

Drenaje biliar internoexterno en paciente con adenocarcinoma de cabeza de páncreas.
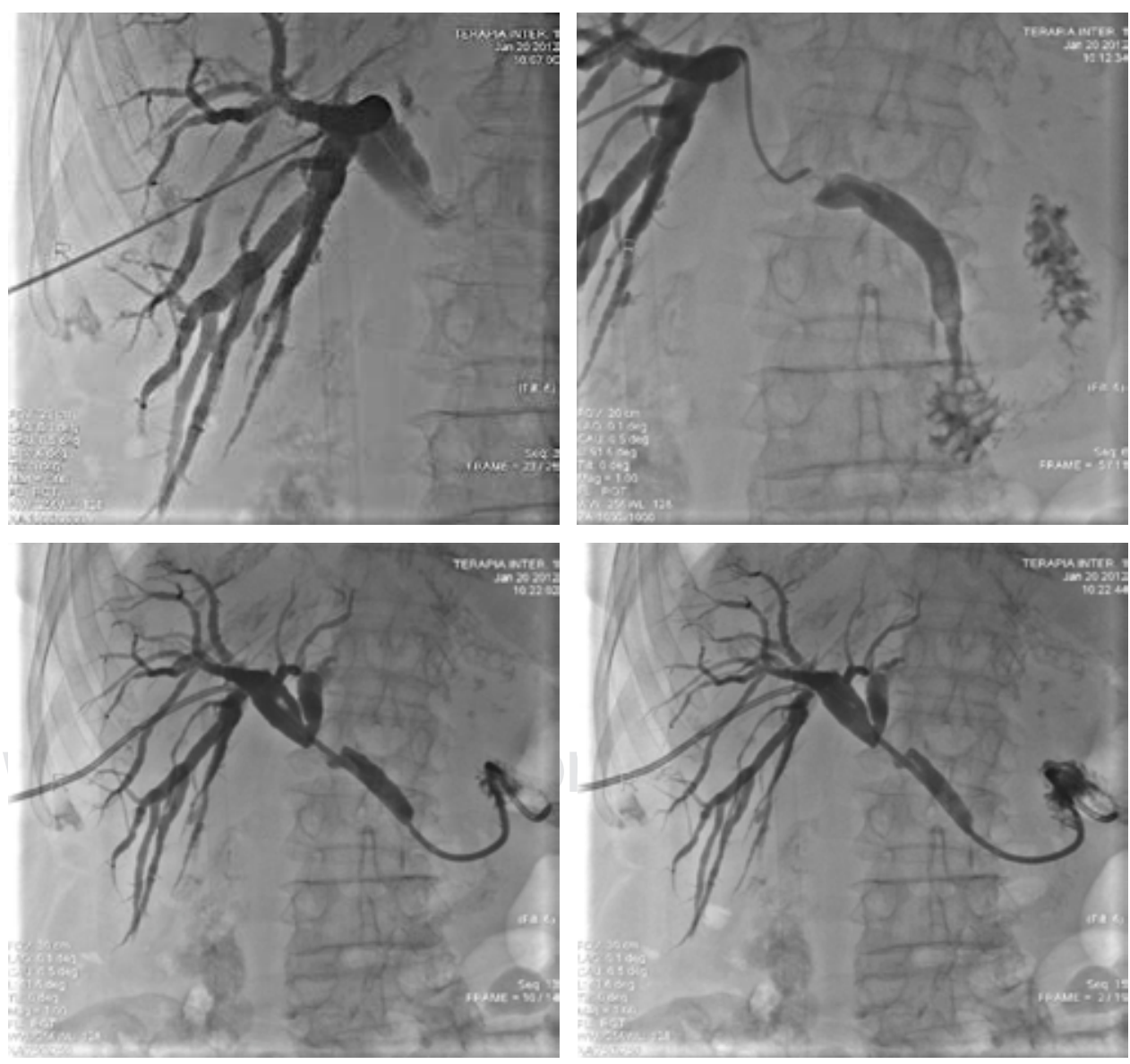

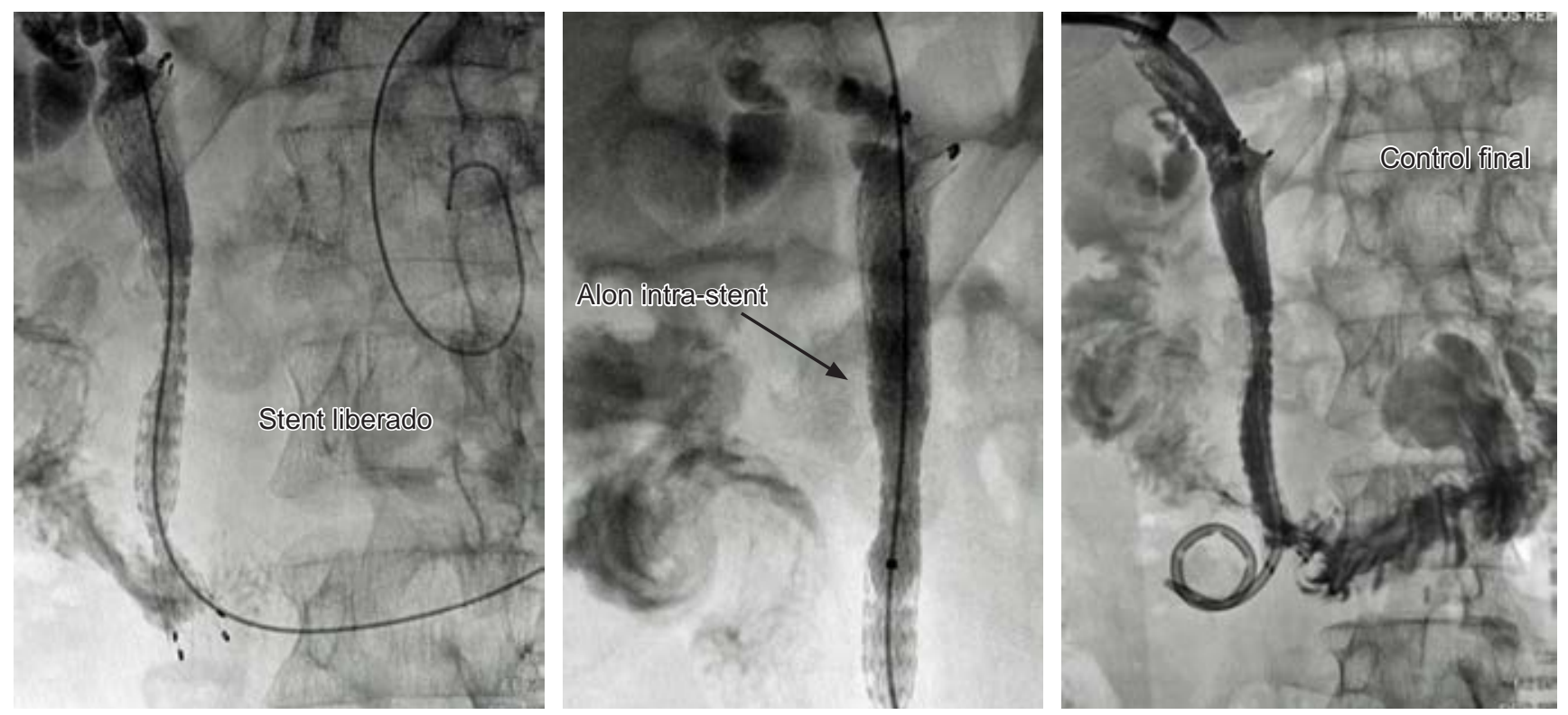

Figura 2: Drenaje biliar interno-externo por adenocarcinoma de páncreas con colocación de endoprótesis metálica.

El material que se utilizó para los procedimientos fue el equipo completo de drenaje biliar Flexima, de la marca Boston Scientific (Boston Scientific Corporation. 300 Boston Scientific Way. Marlborough, MA 01752. USA). En algunos casos, y para tratar de abatir la estenosis, se utilizó una guía hidrofílica Radifocus Guide Wire M 0.035 pulgadas $\times 150$ centímetros, y si se lograba vencer, se avanzaba catéter Radifocus Glidecath Multipurpose $5 \mathrm{Fr}$ con una longitud de 100 centímetros, ambas de la marca Terumo (Terumo Corporation. 44-1, 2-Chome, Hatagaya, Shibuya-Ku, Tokyo, Japan), para posteriormente colocar el catéter de derivación biliar referido, y lograr una derivación externainterna, dejando el extremo distal hasta el duodeno.

En los casos en los que se tuvo que colocar una endoprótesis metálica autoexpandible, se utilizó la endoprótesis E-LUMINEXX (Bard Peripheral Vascular, Inc. 1415 West Third Street. Tempe, Arizona 85281, USA), de diversas medidas, según las dimensiones de la oclusión y/o la estenosis por corregir.

Previa asepsia, se realizó infiltración anestésica en la línea axilar media, bajo control fluoroscópico se visualizó la imagen aérea de duodeno con el propósito de orientación para la ubicación del hilio hepático. Se puncionó con aguja de Chiba 21 por 15 centímetros de longitud, en esa dirección y discretamente en sentido cefálico y anterior hasta obtener bilis.

Una vez logrado esto, se inyectó lentamente y a baja presión para poder ubicar la vía biliar y opacificar sus segmentos intra- y extrahepáticos, realizando la colangiografía percutánea, con el propósito de localizar el sitio de la patología, definiendo si se trataba de estenosis u obstruc- ción total, características de los contornos, posibilidad de identificar causa de la patología, sobre todo en litiasis o en derivaciones biliodigestivas.

A través de la aguja, se introdujo de manera lenta y gentil, bajo visión fluoroscópica, la guía de 0.018 pulgadas hasta que avanzó dentro de la vía biliar. Una vez que se tuvo la certeza de la ubicación de la guía, se retiró lentamente la aguja empleada para la punción, realizando el intercambio por el set de introductor AccuStick, dejando el extremo distal del mismo dentro de la vía biliar, dirigiéndolo hacia el sitio de afectación, ya que a través del introductor 4.5 $\mathrm{Fr}$, una vez retirado el dilatador, se introdujo la guía 0.038 pulgadas $\times 150$ centímetros de acero inoxidable teflonada.

Con esta última guía, se intentó avanzar a través de la zona con estenosis u oclusiva, con el soporte de la misma se introdujeron los dilatadores para piel, tejido celular subcutáneo, musculo y aponeurosis 6 y $8 \mathrm{Fr}$; a continuación, se retiraron éstos, y sobre la misma guía, se ingresó el catéter de derivación biliar 8 Fr de 35 centímetros de longitud, multiperforado, llevándolo hasta el duodeno, entre la segunda y tercera porciones (Figura 1). En ese momento, se decidió el dejar o no la endoprótesis metálica E-LUMINEXX, teniendo en cuenta, sobre todo, el diagnóstico de la ictericia obstructiva, presencia de lesiones metastásicas, y cantidad de canales biliares intrahepáticos comprometidos.

Si no se pudo pasar fácilmente la zona afectada con la guía, se cambió por otra guía hidrofílica Radifocus Guide Wire M 0.035 pulgadas $\times 150$ centímetros, con la que se intentó avanzar; si se lograba, se introdujo entonces un catéter Radifocus Glidecath Multipurpose 5 Fr, avanzando hasta la 
segunda porción del duodeno. Una vez ahí, se cambió la guía hidrofílica por la guía teflonada, y se retiró el catéter referido para entonces colocar el catéter de derivación biliar, y también decidir la colocación de la endoprótesis metálica (Figura 2).

En caso de no poder vencer la obstrucción, se decidió dejar sólo un drenaje externo, empleando un catéter $8 \mathrm{Fr}$ para drenaje de colecciones o de nefrostomía Flexima, de la marca Boston Scientific (Figura 3).

Se realizó un estudio retrospectivo, tipo serie de casos en el que se incluyeron pacientes que durante el periodo enero de 2011 a diciembre de 2017 fueron sometidos a drenaje biliar percutáneo por obstrucción de vía biliar benigna o maligna, obteniéndose de los expedientes información demográfica, manifestaciones clínicas, método diagnóstico, resultados de pruebas paraclínicas, la causa de la obstrucción, la tasa de dilatación exitosa y de complicaciones asociadas con el procedimiento.

Se excluyeron los pacientes con información clínica o paraclínica incompleta. El estudio fue sometido al Comité Local de Bioética e Investigación del Hospital Ángeles Mocel para su aprobación.
El análisis estadístico se realizó en SPSS v. 24 para Mac, en el cual se realizaron pruebas estadísticas descriptivas consistentes en media y desviación estándar para variables cuantitativas, frecuencias y porcentajes para variables cualitativas. No se realizaron pruebas estadísticas inferenciales. Los gráficos se realizaron en el programa GraphPad v.6 para Mac.

\section{RESULTADOS}

Un total de 69 procedimientos tipo DBP fueron realizados en el Hospital Ángeles Mocel durante el periodo de estudio, de los cuales se excluyeron dos casos por falta de información completa en el expediente. De los 67 casos restantes, el $67.2 \%$ eran femeninos. Los grupos de edad con mayor número de casos fueron de 41-50 años y 51-60 años, que representaron el $65.7 \%$ de los casos (Tabla 1).

Las patologías malignas de las vías biliares representaron el $86.6 \%$ de los casos y las benignas el $13.4 \%$. Entre las causas malignas el carcinoma de cabeza de páncreas fue el más común y la obstrucción biliar por causa benigna fue secundaria a estenosis posquirúrgica (Tabla 1).
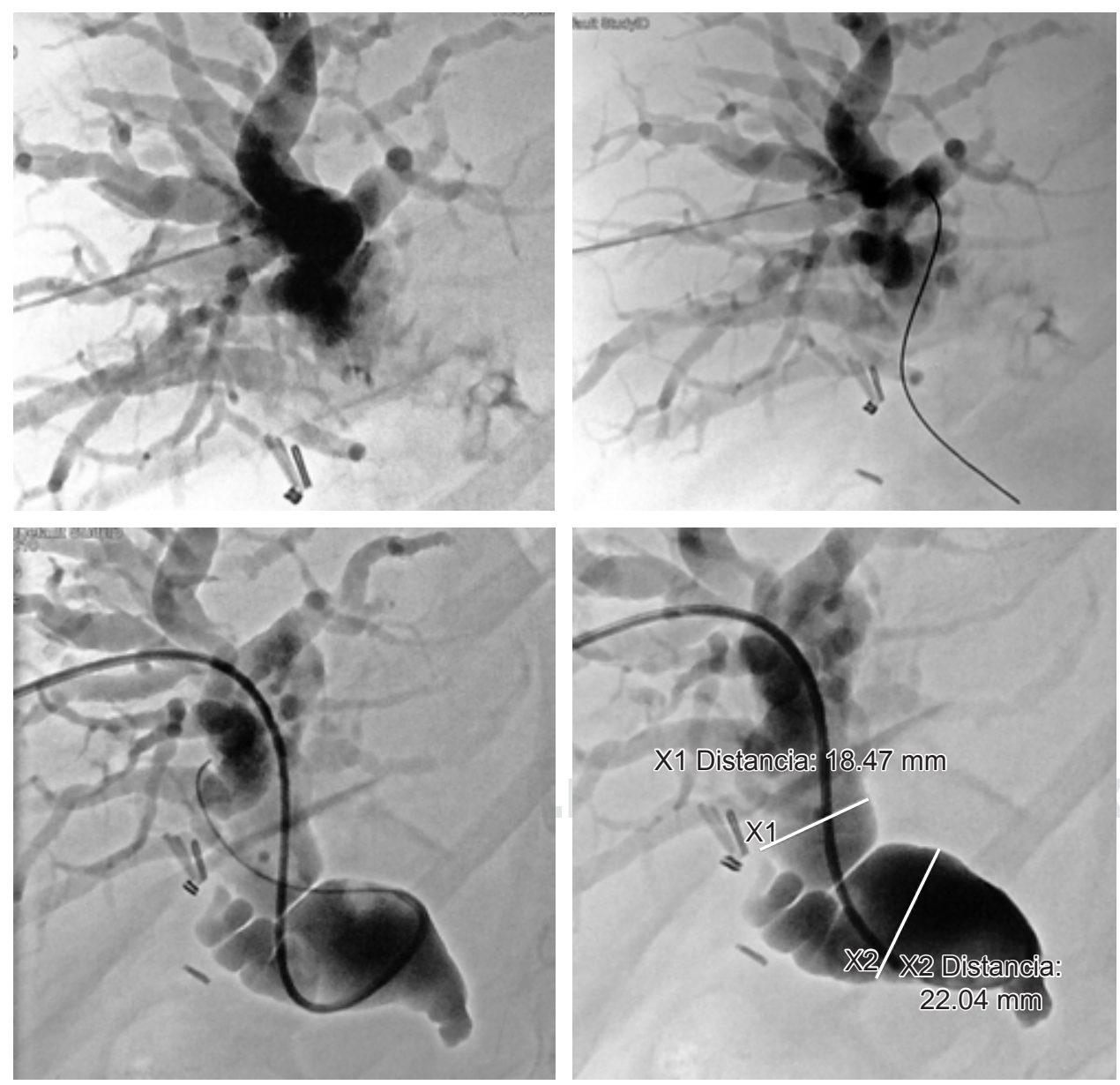

Figura 3:

Drenaje biliar externo en paciente con adenocarcinoma de vías biliares. 
Tabla 1: Características demográficas y causa de la obstrucción biliar de los pacientes.

\begin{tabular}{|c|c|c|}
\hline Característica & $n$ & $\%$ \\
\hline \multicolumn{3}{|l|}{ Grupos de edad (años) } \\
\hline $20-30$ & 6 & 9.0 \\
\hline $31-40$ & 8 & 11.9 \\
\hline $41-50$ & 20 & 29.9 \\
\hline $51-60$ & 24 & 35.8 \\
\hline $61-70$ & 6 & 9.0 \\
\hline$>70$ & 3 & 4.5 \\
\hline \multicolumn{3}{|l|}{ Sexo } \\
\hline Femenino & 45 & 67.2 \\
\hline Masculino & 22 & 32.8 \\
\hline \multicolumn{3}{|l|}{ Causa de la obstrucción biliar } \\
\hline Maligna & 58 & 86.6 \\
\hline $\begin{array}{l}\text { Adenocarcinoma de } \\
\text { cabeza de páncreas }\end{array}$ & 46 & 68.7 \\
\hline Colangiocarcinoma & 9 & 13.4 \\
\hline $\begin{array}{l}\text { Adenocarcinoma de } \\
\text { ámpula de Vater }\end{array}$ & 3 & 4.5 \\
\hline Benigna & 9 & 13.4 \\
\hline Estenosis posquirúrgica & 9 & 13.4 \\
\hline
\end{tabular}

Las manifestaciones clínicas más frecuentes fueron la ictericia (86\%) y la pérdida de peso (78\%), mientras que la fiebre se encontró en la tercera parte de los pacientes. Los valores de exámenes de laboratorio se presentaron con los siguientes rangos como mínimos y máximos respectivamente: bilirrubinas totales (1.5-14.2 $\mathrm{mg} / \mathrm{dL})$, fosfatasa alcalina (167-834 UI/L), TGO (19-125 UI/L), TGP (21-139 UI/L), albúmina (0.1-3.1 g/dL).

Para decidir la estrategia terapéutica apropiada, se definió el grado de obstrucción y las características de la vía biliar, encontrándose una vía biliar dilatada en el $85.1 \%$ de los casos $(n=57)$ y vía biliar normal en el $14.9 \%(n=10)$. Entre los pacientes con vía biliar dilatada $(n=57)$ (Tabla 2).

Ocurrieron complicaciones solamente en tres pacientes de 55 , sometidos exitosamente a DBP, equivalente a una tasa de complicaciones de 5.4\%; dos de éstos desarrollaron sepsis y uno hemorragia que requirió transfusión.

En el seguimiento posterior al procedimiento, la ictericia y síntomas asociados desaparecieron en un promedio de siete días. En quienes presentaban fiebre, ésta desapareció en el transcurso de 24 horas. En el caso de los 12 pacientes a los que se les colocó endoprótesis por estenosis benigna, el seguimiento se ha logrado a más de tres años sin complicaciones posteriores. En el caso de los pacientes con lesiones malignas, la sobrevida a cinco años ha sido de $17.4 \%$.

\section{DISCUSIÓN}

En la presente serie de casos, que resume la experiencia de DBP de siete años en el Hospital Ángeles Mocel, el 96.5\% los pacientes con obstrucción biliar tuvieron un DBP exitoso. Esta alta tasa de éxito es superior a la reportada en la literatura por Parquet y cols., quienes lograron una tasa de éxito de $84 \%$ al primer intento en pacientes con ictericia obstructiva tanto de origen benigno como maligno. ${ }^{11}$ También, la tasa de éxito del presente estudio es superior a la reportada por Choi y colaboradores, quienes tuvieron una tasa de éxito de DBP de $48.4 \%$ en su serie de pacientes con ictericia obstructiva neoplásica. ${ }^{12}$ De modo similar, la tasa de éxito lograda en el Hospital Ángeles Mocel es superior a la reportada por Saluja y equipo, de $89 \%$ en pacientes con cáncer de vesícula biliar. ${ }^{13}$

Por otro lado, esta tasa de éxito es similar a la reportada por Walter y cols., quienes tuvieron una tasa de éxito de $97 \%$ en pacientes con tumor de Klatskin sometidos a DBP, ${ }^{14}$ y ligeramente inferior a la reportada por Kloek en pacientes con sospecha de colangiocarcinoma hiliar. ${ }^{15}$

Entre las complicaciones de DPB reportadas en la literatura se encuentran fugas biliares, bilorrea, hemobilia, colangitis, hemotórax y pancreatitis. ${ }^{16}$ Mientras que, en el presente estudio, las dos complicaciones que ocurrieron fueron sepsis y hemorragia, mismas que fueron tratadas exitosamente; de hecho, no ocurrieron muertes de pacientes asociadas al procedimiento, en contraste con lo reportado por Saluja y equipo, quienes reportaron una mortalidad asociada al procedimiento de $4 \% .{ }^{13}$ Por otro lado, no se presentaron casos de la colangitis en el presente estudio, pero en reportes como el de Walter, se reportó esta complicación en el $21 \%$ de los pacientes. ${ }^{14}$ Por su parte Choi reportó una frecuencia de sangrado de $3.2 \%$, ligeramente superior a la encontrada en el presente estudio. $^{12}$

Tabla 2: Procedimientos realizados y su resultado en los pacientes con vía dilatada.

\begin{tabular}{lrr} 
Procedimientos & $\mathrm{n}$ & $\%$ \\
\hline Procedimientos exitosos & 55 & 96.5 \\
Drenaje externo-interno & 32 & 56.2 \\
Endoprótesis & 12 & 21.1 \\
Drenaje externo & 11 & 19.2 \\
Procedimientos no exitosos & 2 & 3.5 \\
Múltiples adherencias & 1 & 1.7 \\
Lesión de páncreas & 1 & 1.7 \\
\hline n = número de observaciones. & &
\end{tabular}


La frecuencia de sepsis en el presente estudio fue de $3.6 \%$, una frecuencia claramente inferior a la reportada por Ahn, de $42.5 \%$ en pacientes sometidos a DBP por patología maligna de la vía biliar. ${ }^{17}$ Es decir que, aunque la frecuencia de sepsis en este estudio fue baja, representa un área de oportunidad para mejorar aún más los resultados del DBP entre los pacientes del Hospital Ángeles Mocel.

Además de sus fortalezas obvias, el presente trabajo tiene como principal debilidad su naturaleza descriptiva y retrospectiva, así como la falta de registro de mortalidad a 30 días y el modesto tamaño de muestra. Sin embargo, es un buen punto de partida para la realización de estudios posteriores y permite presentar a la comunidad médica la experiencia de realización en el Hospital Ángeles Mocel de DBP y sus complicaciones asociadas.

\section{CONCLUSIONES}

La tasa de éxito de DBP en pacientes del Hospital Ángeles Mocel con ictericia obstructiva por patologías neoplásicas y no neoplásicas se encuentra entre las más altas reportadas en la literatura. Esto se logró por la selección adecuada de los pacientes, por la comunicación estrecha con el médico tratante, así como por los datos obtenidos en los procedimientos que se quisieron realizar por medio de endoscopia, que por algún motivo resultó fallida. Lo anterior fue también condicionante de una baja tasa de complicaciones. Al final del análisis, se demuestra un alto grado de confiabilidad, unido a la experiencia alcanzada en la realización de estos procedimientos.

\section{REFERENCIAS}

1. Balde AK, Balde OT, Barry AB, Camara SN, Sylla H, Diallo AT et al. Obstructive jaundice, study of 33 cases in department of visceral surgery, Donka National Hospital. JCTR. 2016; 4 (2): 16-20.

2. Meseeha M, Attia M. Endoscopic retrograde cholangiopancreatography (ERCP) [Updated 2018 Oct 27]. In: StatPearls [Internet]. Treasure Island (FL): StatPearls Publishing; 2019. Available in: https://www. ncbi.nlm.nih.gov/books/NBK493160/

3. Cotton PB. ERCP Introduction: Developments in ERCP over 40 years. In: Cotton PB, Leung J. Advanced digestive endoscopy: ERCP. 2nd edition. Massachusetts: Blackwell Publishing; 2016. pp. 7-13.
4. Balderramo D, Sendino O, Burrel M, Real MI, Blasi A, Martinez-Palli $\mathrm{G}$ et al. Risk factors and outcomes of failed endoscopic retrograde cholangiopancreatography in liver transplant recipients with anastomotic biliary strictures: a case-control study. Liver Transpl. 2012; 18 (4): 482-489.

5. Niemela J, Kallio R, Ohtonen P, Perala J, Saarnio J, Syrjala H. Is Palliative percutaneous drainage for malignant biliary obstruction useful? World J Surg. 2018; 42 (9): 2980-2986.

6. Artifon EL, Aparicio D, Paione JB, Lo SK, Bordini A, Rabello C et al. Biliary drainage in patients with unresectable, malignant obstruction where ERCP fails: endoscopic ultrasonography-guided choledochoduodenostomy versus percutaneous drainage. J Clin Gastroenterol. 2012; 46 (9): 768-774.

7. Ring EJ, Kerlan RK Jr. Interventional biliary radiology. AJR Am J Roentgenol. 1984; 142 (1): 31-34.

8. Marín J. Intervencionismo biliar en enfermedad benigna. Rev Colomb Gastroent. 2003; 18 (3): 85-94.

9. Téllez-Ávila FI, Herrera-Mora D, Duarte-Medrano G, Lopez-Arce G, Lindoro-Barraza D, Casanova I et al. Biliary drainage in patients with failed ercp: percutaneous versus EUS-guided drainage. Surg LaparosC Endosc Percutan Tech. 2018; 28 (3): 183-187.

10. Günther RW, Schild H, Thelen M. Percutaneous transhepatic biliary drainage: experience with 311 procedures. Cardiovasc Intervent Radiol. 1988; 11 (2): 65-71.

11. Parquet VG, Ortiz BS, Samaniego AC, Giménez VA. Drenaje biliar percutáneo: técnica, indicaciones y resultados. An Fac Cienc Méd (Asunción). 2005; 38 (3): 61-67.

12. Choi J, Ryu JK, Lee SH, Ahn DW, Hwang JH, Kim YT et al. Biliary drainage for obstructive jaundice caused by unresectable hepatocellular carcinoma: the endoscopic versus percutaneous approach. Hepatobiliary Pancreat Dis Int. 2012; 11 (6): 636-642.

13. Saluja SS, Gulati M, Garg PK, Pal H, Pal S, Sahni P et al. Endoscopic or percutaneous biliary drainage for gallbladder cancer: a randomized trial and quality of life assessment. Clin Gastroenterol Hepatol. 2008; 6 (8): 944-950.e3.

14. Walter T, Ho CS, Horgan AM, Warkentin A, Gallinger S, Greig PD et al. Endoscopic or percutaneous biliary drainage for Klatskin tumors? J Vasc Interv Radiol. 2013; 24 (1): 113-121.

15. Kloek JJ, van der Gaag NA, Aziz Y, Rauws EA, van Delden OM, Lameris JS et al. Endoscopic and percutaneous preoperative biliary drainage in patients with suspected hilar cholangiocarcinoma. J Gastrointest Surg. 2010; 14 (1): 119-125.

16. Wagner A, Mayr C, Kiesslich T, Berr F, Friesenbichler P, Wolkersdörfer GW. Reduced complication rates of percutaneous transhepatic biliary drainage with ultrasound guidance. J Clin Ultrasound. 2017; 45 (7): 400-407.

17. Ahn S, Lee YS, Lim KS, Lee JL. Malignant biliary obstructions: can we predict immediate postprocedural cholangitis after percutaneous biliary drainage? Support Care Cancer. 2013; 21 (8): 2321-2316. 